\title{
A Case of primary adrenal lymphoma presenting with adrenal insufficiency
}

Samarasinghe GP', Antonypillai CN¹, Kalubowila UP', Ratnayake RMP1, Ekanayake EMSB', Thilakarathne BMIK ${ }^{1}$

${ }^{1}$ Teaching Hospital Kandy

\section{Abstract}

Non-Hodgkin lymphoma (NHL) with secondary involvement of the adrenal gland is not uncommon, however primary adrenal lymphoma leading to adrenal insufficiency is a rare occurrence. We report a case of primary adrenal lymphoma (PAL) that presented with adrenal insufficiency. Presence of persistent hyponatremia lead to the diagnosis of adrenal insufficiency. Computerized Tomography revealed the presence of bilateral large adrenal masses without lymphadenopathy or other organ involvement and histology confirmed the diagnosis of diffuse Non-Hodgkin B Cell Lymphoma. He was given hydrocortisone replacement therapy and was referred for chemotherapy though he died due to the poor prognosis of this malignancy.

Key words: Non-Hodgkin lymphoma, adrenal insufficiency, adrenal mass.

DOI: http://doi.org/10.4038/sjdem.v8i1.7350

Received:15 $5^{\text {th }}$ December 2017

Accepted revised version: 1 st March 2018

Published:9th April 2018

Correspondence: e-mail: gayanipramuditha81@gmail.com

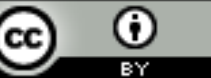

This is an open-access article distributed under the terms of the Creative Commons Attribution 4.0 International License, which permits unrestricted use, distribution and reproduction in any medium provided the original author and source are credited 


\section{Introduction}

Non-Hodgkin lymphomas (NHL) can arise from almost any organ in the body other than the lymph nodes $(1,2)$. However primary endocrine lymphomas accounts for only 3 percent of all extra nodal lymphomas and thyroid and testis are the commonest sites to be involved. Management of extra nodal lymphomas could be challenging due to the presence of organ specific complications. Secondary involvement of the adrenal gland is not uncommon, having the prevalence of $4 \%$ while primary adrenal lymphoma is extremely rare (3).

Primary adrenal lymphomas (PALs) are typically bilateral and the patients may present with or without features of adrenal insufficiency (4). Majority are males in their sixth or seventh decade of life (5). Some patients have large bilateral adrenal masses and image guided biopsy for histology is a crucial investigation to confirm the diagnosis provided it is not Phaeochromocytoma. The prognosis is generally considered to be poor owing to the tumor aggressiveness and the rapid progression (3).

Case

A 56year old male was admitted to the Gastroenterology unit, Teaching Hospital Kandy with a history of intermittent generalized abdominal pain, loss of appetite and loss of weight for 1-month. He had intermittent diarrhea without blood or mucus in the stools. He also complained of a one-month history of low grade intermittent fever for which he was investigated as for pyrexia of unknown origin.

He did not have a cough, hemoptysis or shortness of breath. Further questioning revealed body weakness, easy fatigability and recurrent faintishness which was aggravated by upright posture. He did not have excessive sweating, palpitations or anxiety. He did not have a history of tuberculosis or any autoimmune diseases. He had been a diabetic for the past 14 years with poor control and had been on premixed insulin for the last 4years.

However, he had stopped taking insulin due to recurrent hypoglycemic events and continued only with metformin in the recent past with a glycosylated haemoglobin level (HbA1C) of $8.1 \%$ showing a satisfactory control. He was a nonsmoker and denied illicit drug abuse. On examination he looked ill and was pale but had no crease, scar or buccal pigmentation. There was no peripheral lymphadenopathy. Pulse rate was 90 beats per minute and the blood pressure was $90 / 70 \mathrm{mmHg}$ with a significant postural drop. Rest of the cardiovascular system and respiratory system examination was clinically normal. Abdominal examination did not reveal hepatosplenomegaly. On admission he was resuscitated with intravenous fluids to maintain his blood pressure in the normal range. The laboratory work- up revealed persistent hyponatremia with serum sodium $121 \mathrm{mmol} / 1$ (135-145), serum potassium $5.7 \mathrm{mmol} / 1$ (3.5-5) on admission. Random blood glucose was $88 \mathrm{mg} / \mathrm{dl}$. Other biochemical and hematological investigations are as follows (Table 1).

He continued to have low blood pressure with postural symptoms associated with persistent hyponatremia indicating the possibility of cortisol insufficiency. He was treated with intravenous hydrocortisone after taking blood for random cortisol level which was $105 \mathrm{nmol} / 1$ (100-650). Short synacthen test results showed a basal 9 am cortisol of $97 \mathrm{nmol} / 1$ with $218,405 \mathrm{nmo} / 1$ after 30 minutes and 1 hour after synthetic ACTH administration, suggesting a sub optimal cortisol response. ACTH of 49pmol/1 (2-10) suggested primary adrenal insufficiency. X-ray abdomen did not show adrenal calcification.

Mantoux test was negative for evidence of tuberculosis infection and the chest X-ray was normal with no lymphadenopathy. Contrast enhanced Computerized Tomography (CT) of chest and abdomen revealed bilateral solid adrenal masses, right measured $62 \mathrm{x}$ $88 \times 70 \mathrm{~mm}$ and left measured 61x 69x 70mm. There was no hepatosplenomegaly. Mild non-significant enlargement $(<10 \mathrm{~mm})$ of the paraaortic lymph nodes were noted. Visualized lung fields were normal without mediastinal lymphadenopathy.

The possibility of phaeochromocytoma was excluded by the normal 24-hour urine metanephrine level, 65ng/l (0170). Subsequently he underwent CT guided biopsy of the right adrenal mass and histology revealed an extensively necrotic tumor containing focal infiltrate of monomorphic population of cells with hyperchromatic nuclei and scanty cytoplasm. Immunohistochemistry confirmed the diagnosis of $\mathrm{B}$ cell non-Hodgkin lymphoma by the expression of LCA and CD 20. CD3 was negative and proliferation index was $>40 \%$.

Colonoscopy was done to evaluate prominent lower intestinal symptoms, which did not show features of intestinal lymphoma. Bone marrow biopsy was negative for tumor cells. 


\section{Table 1: Laboratory investigations}

Test

Blood urea

Serum Creatinine

Full blood count

$\mathrm{Hb} \%$

White cell count

Platelet

ESR

CRP

AST

ALT

Alkaline phosphatase

Lactate dehydrogenase

Serum protein

Serum albumin

HbA1C
Results

$8.5 \mathrm{mmol} / 1$

$97 \mathrm{micromol} / 1$

62-106

$11-16$

$3-10$

$150-450$

$48 \mathrm{~mm} / 1$

5-30

$147 \mathrm{mg} / 1$

$<10$

$42 \mathrm{U} / 1$

5-45

5-40

40-129

240-480

6-8

$3.2-4.5$

$<7 \%$

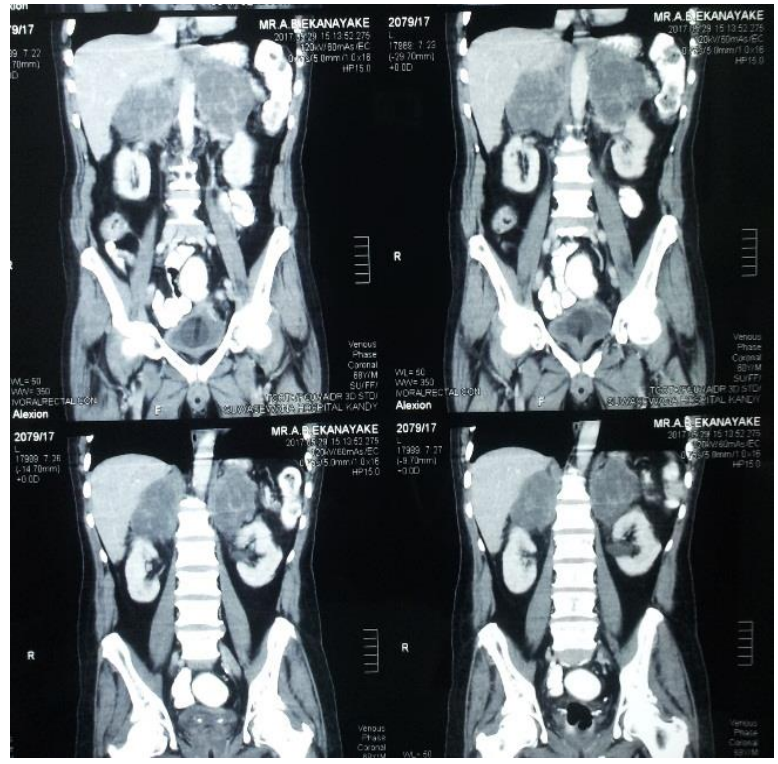

The patient was referred to the Oncology unit for chemotherapy and there he was treated with R-CHOP regimen (Rituximab - cyclophosphamide, doxorubicin, vincristine and prednisone). Intravenous hydrocortisone injections were continued. Unfortunately, the patient died 2 weeks after starting chemotherapy.

Figure 1: CT abdomen showing large bilateral adrenal masses. 


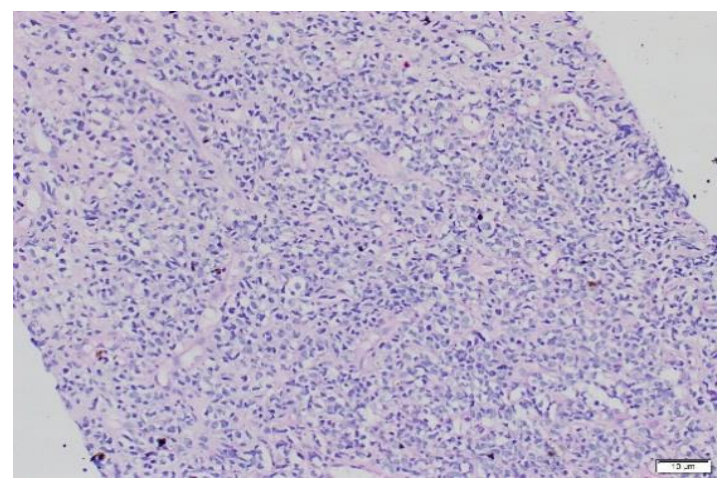

Figure 2: H \& E stain showing monomorphic cell infiltration

\section{Discussion}

Addison's disease or primary adrenal insufficiency is commonly due to autoimmune destruction of the adrenal glands, adrenal tuberculosis or idiopathic causes. A study done in south Africa has shown that malignancy accounts for only $6 \%$ of the cases (6). Involvement of the adrenal glands in malignancy could even be commoner than expected since many of the patient's die of the tumor before the diagnosis of Addison's disease is made. Renal cell carcinoma, melanoma, lung carcinoma, breast carcinomas are known to metastasize to the adrenals while lymphoma can infiltrate the gland as secondary deposits or as a primary lesion (7).

Primary adrenal lymphoma is a rare disease of the adrenal gland with only 200 reported cases worldwide, where high degree of clinical suspicion is required for the diagnosis to start on specific treatment (8). Nonspecific symptoms and the absence of typical finding of a lymphoma make the diagnosis difficult. It is defined as adrenal lymphoma without evidence of lymphoma elsewhere or unequivocally dominant adrenal lesions if lymph nodes or other organs are involved (9). Extra nodal presentation accounts for approximately one third of NHL cases, which commonly arises from the spleen, bone marrow, skin, small intestine, stomach and brain (1). The etiology and pathogenesis of primary adrenal lymphoma is not clear. However immune dysfunction occurring in haematopoietic cells in the adrenal glands, Epstein barr and other viral infections, mutations in the p53 and c-kit genes are some of the proposed mechanisms causing this tumor $(1,10)$. PAL typically occurs in elderly males and the average age at diagnosis is 70 years $(9,11)$. Majority has bilateral involvement and the incidence of adrenal

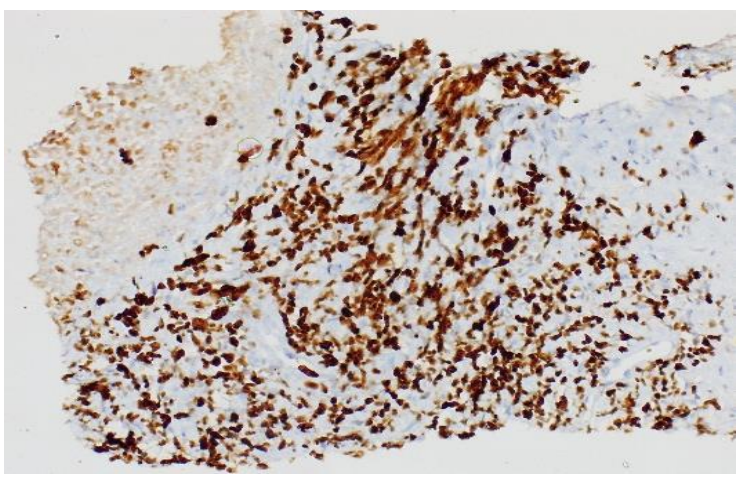

Figure 3: Immunostaining for CD20 positive B Cell Non-Hodgkin lymphoma

insufficiency is $61 \%$ which is significantly higher in comparison to other metastatic tumors $(4,9)$. Patients may present with weight loss, fatigue, hypotension or pigmentation; however, the diagnosis of adrenal failure can easily be overlooked as these overlap with the lymphoma associated B symptoms. There are reported cases of PAL developing adrenal insufficiency during the course of chemotherapy. The reason for adrenocortical insufficiency is believed to be due to either direct invasion of the tumor or cytokine driven functional paracrine effect caused by the lymphoma cells. The latter is explained by the high frequency of adrenal insufficiency compared to the other malignancies (9).

Adrenal lymphoma appears on CT as discrete masses or diffuse gland involvement while the shape of the gland is maintained (12). CT guided biopsy of the lesion after excluding the possibility of pheochromocytoma, confirms the diagnosis. The commonest histological type is diffuse large B cell lymphoma accounting for $86 \%$ of the cases (13). Commonly used current treatment regimen is R-CHOP (Rituximab, Cyclophosphamide, Doxorubicin, Vincristine and Prednisone). Despite the use of advanced chemotherapeutic regime, PAL carries a poor prognosis.

\section{Conclusion}

PAL is a rare cause of primary adrenal insufficiency. High degree of suspicion is required for the diagnosis. Glucocorticoid replacement should be continued even during chemotherapy to prevent life threatening adrenal crisis. Despite advanced chemotherapy it is associated with poor prognosis. 
1. Zucca E, Extranodal lymphoma: a reappraisal. Ann Oncol, 2008. 19 Suppl 4: p. iv77-80.

2. Mertsoylu H, et al, Primary extranodal non-Hodgkin's lymphoma: clinicopathological features, survival and treatment outcome in two cancer centers of southern Turkey. Asian Pac J Cancer Prev, 2014. 15(17): p. 7207-11.

3. Freeman C, Berg J W, Cutler S J. Occurrence and prognosis of extranodal lymphomas. Cancer, 1972. 29(1): p. 25260.

4. Rashidi A, Fisher S I. Primary adrenal lymphoma: a systematic review. Ann Hematol, 2013. 92(12): p. $1583-93$.

5. Laurent $\mathrm{C}$ et al. Adrenal lymphoma: presentation, management and prognosis. Qjm, 2017. 110(2): p. $103-109$.

6. Soule S, Addison's disease in Africa--a teaching hospital experience. Clin Endocrinol (Oxf), 1999. 50(1): p. 115-20.

7. Mor F et al. Addison's disease due to metastases to the adrenal glands. Postgrad Med J, 1985. 61(717): p. 637-9.

8. Connors, J M. Non-Hodgkin lymphoma: the clinician's perspective--a view from the receiving end. Mod Pathol, 2013. 26 Suppl 1: p. S111-8.

9. Harada $\mathrm{K}$ et al. The Clinical and Hormonal Characteristics of Primary Adrenal Lymphomas: The Necessity of Early Detection of Adrenal Insufficiency. Intern Med, 2017. 56(17): p. 2261-2269.

10. Ohsawa, $\mathrm{M}$ et al. Malignant lymphoma of the adrenal gland: its possible correlation with the Epstein-Barr virus. Mod Pathol, 1996. 9(5): p. 534-43.

11. Chen P et al. Bilateral primary adrenal diffuse large B cell lymphoma without adrenal insufficiency: A case report and review of the literature. Mol Clin Oncol, 2017. 7(1): p. 145-147.

12. Dunnick N R, Korobkin M, Imaging of adrenal incidentalomas: current status. AJR Am J Roentgenol, 2002. 179(3): p. 559-68.

13. Kim Y R et al. Prognostic factors in primary diffuse large B-cell lymphoma of adrenal gland treated with rituximabCHOP chemotherapy from the Consortium for Improving Survival of Lymphoma (CISL). J Hematol Oncol, 2012. 5: p. 49. 